\title{
A proof of a conjecture on the distance spectral radius and maximum transmission of graphs
}

\author{
Lele Liu*, Haiying Shan ${ }^{\dagger}, \quad$ Changxiang He ${ }^{\ddagger}$
}

\begin{abstract}
Let $G$ be a simple connected graph, and $D(G)$ be the distance matrix of $G$. Suppose that $D_{\max }(G)$ and $\lambda_{1}(G)$ are the maximum row sum and the spectral radius of $D(G)$, respectively. In this paper, we give a lower bound for $D_{\max }(G)-\lambda_{1}(G)$, and characterize the extremal graphs attaining the bound. As a corollary, we solve a conjecture posed by Liu, Shu and Xue.
\end{abstract}

Keywords: Distance matrix; Distance spectral radius; Non-transmission-regular graph AMS Classification: $05 \mathrm{C} 50$

\section{Introduction}

In this paper, we consider only simple, undirected graphs, i.e, undirected graphs without multiple edges or loops. A graph $G$ is a pair $(V(G), E(G))$ consisting of a set $V(G)$ of vertices and a set $E(G)$ of edges. The distance matrix $D(G)=\left(d_{i j}\right)$ of a connected graph $G$ is the $|V(G)| \times|V(G)|$ matrix indexed by the vertices of $G$, where $d_{i j}=d\left(v_{i}, v_{j}\right)$ and $d\left(v_{i}, v_{j}\right)$ denotes the distance between the vertices $v_{i}$ and $v_{j}$, i.e., the length of a shortest path between $v_{i}$ and $v_{j}$. The largest eigenvalue of $D(G)$, denoted by $\lambda_{1}(G)$, is called the distance spectral radius of $G$. For graph notation and terminology undefined here we refer the reader to [2].

The transmission of a vertex $v$ in $G$, denoted by $D_{v}(G)$ or simply by $D_{v}$, is the sum of the distances from $v$ to all other vertices in $G$. We denote the maximum and minimum transmission of $G$ by $D_{\max }$ and $D_{\min }$, respectively. That is,

$$
D_{\max }:=\max \left\{D_{v} \mid v \in V(G)\right\}, D_{\min }:=\min \left\{D_{v} \mid v \in V(G)\right\} .
$$

If there is no risk of confusion we shall denote $D_{\max }(G)$ and $D_{\min }(G)$ simply by $D_{\max }$ and $D_{\min }$, respectively. A connected graph $G$ is transmission-regular if $D_{\max }=D_{\min }$; otherwise, $G$ is non-transmission-regular. The Wiener index $W(G)$ of a graph $G$ is the sum of the distances

\footnotetext{
${ }^{*}$ College of Science, University of Shanghai for Science and Technology, Shanghai 200093, China (ahhylau@outlook.com)

${ }^{\dagger}$ School of Mathematical Sciences, Tongji University, Shanghai 200092, China (shan_haiying@tongji.edu.cn)

${ }^{\ddagger}$ College of Science, University of Shanghai for Science and Technology, Shanghai 200093, China (changxiang-he@163.com)
} 
between all unordered pairs of vertices of $G$. Alternatively, $W(G)$ is half the sum of all the entries of the distance matrix of $G$, that is,

$$
W(G)=\frac{1}{2} \sum_{v \in V(G)} D_{v} .
$$

In [1], Atik and Panigrahi introduced a class of graphs called DVDR graphs. A connected graph $G$ on $n$ vertices is said to be distinguished vertex deleted regular graph (DVDR) if there exist a vertex $v$ in $G$ such that the degree $d(v)=n-1$ and $G-v$ is an regular graph. The vertex $v$ is said to be a distinguished vertex of the DVDR graph $G$. If $G-v$ is $r$-regular, we say $G$ is an $r-D V D R$ graph.

In [3], Liu, Shu and Xue studied the bounds of $D_{\max }(G)-\lambda_{1}(G)$ for connected nontransmission-regular graphs, and posed the following conjecture.

Conjecture $1.1([3])$. Let $G$ be a connected non-transmission-regular graph with $n$ vertices. Then

$$
D_{\max }(G)-\lambda_{1}(G)>\frac{1}{n+1} .
$$

It is shown that the conjecture holds for trees in [3]. In this paper, we confirm Conjecture 1.1 by proving the following strengthened results.

Theorem 1.1. Let $G$ be a connected non-transmission-regular graph of order $n$.

(1) If $n$ is odd, then

$$
D_{\max }(G)-\lambda_{1}(G) \geq \frac{n+1-\sqrt{(n-1)(n+3)}}{2} .
$$

Equality holds if and only if $G \cong K_{1,2, \ldots, 2}$.

(2) If $n$ is even, then

$$
D_{\max }(G)-\lambda_{1}(G) \geq \frac{n+2-\sqrt{n^{2}+4 n-4}}{2} .
$$

Equality holds if and only if $G$ is isomorphic to some $(n-4)$-DVDR graph.

\section{Proof of Theorem 1.1}

For a connected graph $G$ of order $n$, write $\sigma(G)$ for the difference between the maximum transmission and the distance spectral radius of $G$, i.e.,

$$
\sigma(G):=D_{\max }(G)-\lambda_{1}(G) .
$$

In this section, we assume $G^{*}$ is a graph attaining the minimum of $\sigma(G)$ among connected non-transmission-regular graphs $G$ of order $n$. Let $\mathbf{x}$ be the positive unit eigenvector of $D\left(G^{*}\right)$ corresponding to $\lambda_{1}\left(G^{*}\right)$. For convenience, we denote $x_{\max }:=\max \left\{x_{v} \mid v \in V\left(G^{*}\right)\right\}$, 
$x_{\min }:=\min \left\{x_{v} \mid v \in V\left(G^{*}\right)\right\}$. Suppose that $D_{\max }$ and $W$ are the maximum transmission and Wiener index of $G^{*}$, respectively. An easy calculation implies that

$$
\begin{aligned}
D_{\max }-\lambda_{1}\left(G^{*}\right) & =D_{\max }-2 \sum_{\{u, v\} \subseteq V(G)} d(u, v) x_{u} x_{v} \\
& =\sum_{u \in V(G)}\left(D_{\max }-D_{u}\right) x_{u}^{2}+\sum_{\{u, v\} \subseteq V(G)} d(u, v)\left(x_{u}-x_{v}\right)^{2} \\
& \geq\left(n D_{\max }-2 W\right) x_{\min }^{2}+\sum_{\{u, v\} \subseteq V(G)} d(u, v)\left(x_{u}-x_{v}\right)^{2} .
\end{aligned}
$$

Before continuing the proof of Theorem 1.1, we need the following two results.

Lemma 2.1. Let $K_{1,2, \ldots, 2}$ be the complete $r$-partite graph on $n$ vertices with $n=2 r-1$. Then

$$
\sigma\left(K_{1,2, \ldots, 2}\right)=\frac{n+1-\sqrt{(n-1)(n+3)}}{2} .
$$

Proof. Let $v$ be the distinguished vertex of $K_{1,2, \ldots, 2}$. It is clear that the quotient matrix of $D\left(K_{1,2, \ldots, 2}\right)$ with respect to the equitable partition $\{v\} \cup\left(V\left(K_{1,2, \ldots, 2}\right) \backslash\{v\}\right)$ is

$$
Q=\left[\begin{array}{ll}
0 & n-1 \\
1 & n-1
\end{array}\right] .
$$

As a consequence, $\lambda_{1}\left(K_{1,2, \ldots, 2}\right)$ is equal to the largest eigenvalue of $Q$, i.e.,

$$
\lambda_{1}\left(K_{1,2, \ldots, 2}\right)=\frac{n-1+\sqrt{(n-1)(n+3)}}{2} .
$$

Clearly, $D_{\max }\left(K_{1,2, \ldots, 2}\right)=n$, we immediately obtain

$$
\sigma\left(K_{1,2, \ldots, 2}\right)=D_{\max }\left(K_{1,2, \ldots, 2}\right)-\lambda_{1}\left(K_{1,2, \ldots, 2}\right)=\frac{n+1-\sqrt{(n-1)(n+3)}}{2}
$$

completing the proof.

Lemma 2.2. Let $G$ be an $(n-4)-D V D R$ graph on $n$ vertices. Then

$$
\sigma(G)=\frac{n+2-\sqrt{n^{2}+4 n-4}}{2} .
$$

Proof. Let $v$ be the distinguished vertex of $G$. Then the quotient matrix of $D(G)$ with respect to the equitable partition $\{v\} \cup(V(G) \backslash\{v\})$ is

$$
\left[\begin{array}{cc}
0 & n-1 \\
1 & n
\end{array}\right]
$$

Therefore, we obtain that

$$
\lambda_{1}(G)=\frac{n+\sqrt{n^{2}+4 n-4}}{2} .
$$


Clearly, $D_{\max }(G)=n+1$, we have

$$
\sigma(G)=D_{\max }(G)-\lambda_{1}(G)=\frac{n+2-\sqrt{n^{2}+4 n-4}}{2}
$$

completing the proof.

In the following, we denote

$$
\sigma_{n}:=\frac{n+\gamma_{n}+\sqrt{\left(n+\gamma_{n}\right)^{2}-4 \gamma_{n}}}{2},
$$

where $\gamma_{n}=1$ if $n$ is odd; $\gamma_{n}=2$ if $n$ is even. By simple algebra, we see

$$
\sigma_{n}^{2}-\left(n+\gamma_{n}\right) \sigma_{n}+\gamma_{n}=0 .
$$

It is obvious that if $n$ is odd, then $\sigma_{n}=\sigma\left(K_{1,2, \ldots, 2}\right)$; if $n$ is even, then $\sigma_{n}=\sigma(G)$ for any $(n-4)$-DVDR graph $G$. Noting that $K_{1,2, \ldots, 2}$ and each $(n-4)$-DVDR graph are both connected non-transmission-regular graphs, we see $\sigma\left(G^{*}\right) \leq \sigma_{n}$.

Proof of Theorem 1.1. Let $u$ and $v$ be two vertices such that $x_{u}=x_{\max }$ and $x_{v}=x_{\min }$, respectively. For short, write $\lambda_{1}:=\lambda_{1}\left(G^{*}\right)$. Using equation $D\left(G^{*}\right) \mathbf{x}=\lambda_{1} \mathbf{x}$ with respect to vertex $u$ we see

$$
\begin{aligned}
\lambda_{1} x_{u} & =\sum_{w \in V\left(G^{*}\right)} d(u, w) x_{w} \\
& =\sum_{w \in V\left(G^{*}\right) \backslash\{v\}} d(u, w) x_{w}+d(u, v) x_{v} \\
& \leq\left(D_{\max }-d(u, v)\right) x_{u}+d(u, v) x_{v}
\end{aligned}
$$

which yields that

$$
D_{\max }-\lambda_{1} \geq d(u, v)\left(1-\frac{x_{v}}{x_{u}}\right) \geq 1-\frac{x_{v}}{x_{u}} .
$$

On the other hand, $\sigma\left(G^{*}\right)=D_{\max }-\lambda_{1} \leq \sigma_{n}$. Hence, we obtain

$$
\frac{x_{\max }}{x_{\min }}=\frac{x_{u}}{x_{v}} \leq \frac{1}{1-\sigma_{n}} .
$$

Our proof hinges on the following five claims.

Claim 1. $n D_{\max }-2 W=\gamma_{n}$.

Proof of Claim 1. We prove this claim by contradiction. Let $n$ be odd, from (2.1) and (2.3) we have

$$
D_{\max }-\lambda_{1} \geq 2 x_{\min }^{2} \geq 2\left(1-\sigma_{n}\right)^{2} x_{\max }^{2}>\frac{2\left(1-\sigma_{n}\right)^{2}}{n}>\sigma_{n},
$$

the last inequality follows from $\sigma_{n}^{2}-(n+1) \sigma_{n}+1=0$. This is a contradiction with $D_{\max }-\lambda_{1} \leq$ $\sigma_{n}$. 
Let $n$ be even. Noting that $2 \mid\left(n D_{\max }-2 W\right)$, we have $n D_{\max }-2 W \geq 4$. It follows from (2.1) and (2.3) that

$$
D_{\max }-\lambda_{1} \geq 4 x_{\min }^{2} \geq 4\left(1-\sigma_{n}\right)^{2} x_{\max }^{2}>\frac{4\left(1-\sigma_{n}\right)^{2}}{n}>\sigma_{n},
$$

a contradiction.

Claim 2. $D_{u}>D_{\min }$ and $D_{v}<D_{\max }$.

Proof of Claim 2. By equation $D\left(G^{*}\right) \mathbf{x}=\lambda_{1} \mathbf{x}$ with respect to vertex $u$ we have

$$
\lambda_{1} x_{u}=\sum_{w \in V\left(G^{*}\right)} d(w, u) x_{w} \leq D_{u} x_{u}
$$

which implies $\lambda_{1} \leq D_{u}$. Since $\lambda_{1}>D_{\min }$, we obtain $D_{u}>D_{\min }$. Similarly, we can prove that $D_{v}<D_{\max }$, completing the proof of this claim.

Claim 3. $\frac{x_{\max }}{x_{\min }}=\frac{1}{1-\sigma_{n}}, D_{\max }-\lambda_{1}=\sigma_{n}$, and there are $(n-1)$ vertices attaining $x_{\max }$.

Proof of Claim 3. Since $D\left(G^{*}\right) \mathbf{x}=\lambda_{1} \mathbf{x}$, we have $\lambda_{1} \mathbf{1} \mathbf{x}=\mathbf{1} D\left(G^{*}\right) \mathbf{x}$, where $\mathbf{1}$ is the all-ones vector of dimension $n$. Hence,

$$
\lambda_{1} \sum_{w \in V\left(G^{*}\right)} x_{w}=\sum_{w \in V\left(G^{*}\right)} D_{w} x_{w} .
$$

It follows from Claim 1 that

$$
\begin{aligned}
\left(D_{\max }-\lambda_{1}\right) \sum_{w \in V\left(G^{*}\right)} x_{w} & =\sum_{w \in V\left(G^{*}\right)}\left(D_{\max }-D_{w}\right) x_{w} \\
& \geq\left(n D_{\max }-2 W\right) x_{\min } \\
& =\gamma_{n} x_{\min } .
\end{aligned}
$$

Therefore we have

$$
D_{\max }-\lambda_{1} \geq \frac{\gamma_{n} x_{\min }}{\sum_{w \in V\left(G^{*}\right)} x_{w}} \geq \frac{\gamma_{n} x_{\min }}{x_{\min }+(n-1) x_{\max }} .
$$

Since $D_{\max }-\lambda_{1} \leq \sigma_{n}$, we find that

$$
\frac{x_{\max }}{x_{\min }} \geq \frac{\gamma_{n} / \sigma_{n}-1}{n-1}
$$

On the other hand, by (2.3) we have

$$
\frac{x_{\max }}{x_{\min }} \leq \frac{1}{1-\sigma_{n}} .
$$

In view of (2.2) we see

$$
\left(\frac{\gamma_{n}}{\sigma_{n}}-1\right)\left(1-\sigma_{n}\right)=\frac{\gamma_{n}}{\sigma_{n}}+\sigma_{n}-\gamma_{n}-1=n-1,
$$


which yields that

$$
\frac{\gamma_{n} / \sigma_{n}-1}{n-1}=\frac{1}{1-\sigma_{n}}
$$

Hence, all inequalities above must be equalities, we immediately obtain $x_{\max } / x_{\min }=(1-$ $\left.\sigma_{n}\right)^{-1}$. Noting that $D_{\max }-\lambda_{1} \leq \sigma_{n}$, together with (2.4), we get $D_{\max }-\lambda_{1}=\sigma_{n}$, completing the proof of this claim.

Claim 4. Let $S=\left\{w \in V\left(G^{*}\right): D_{w}=D_{\max }\right\}$. Then $|S|=n-1$.

Proof of Claim 4. If $n$ is odd, the result follows from Claim 1 immediately. If $n$ is even, then $|S| \in\{n-2, n-1\}$ by Claim 1 . In view of Claim 3, there are $(n-1)$ vertices attaining $x_{\max }$. Hence, if $|S|=n-2$, there exists a vertex $w$ such that $x_{w}=x_{\max }$, while $D_{w}=D_{\max }-1=D_{\min }$, which is a contradiction with Claim 2. Hence, $|S|=n-1$, as required.

Claim 5. $D_{\max }=n+\gamma_{n}-1$ and $D_{\min }=n-1$.

Proof of Claim 5. According to Claim 3, there are $(n-1)$ vertices attaining $x_{\max }$, and the remaining vertex $v$ attaining $x_{\min }$. By Claim 2, $D_{v}<D_{\max }$. Together with Claim 4 we have $D_{v}=D_{\max }-\gamma_{n}=D_{\min }$. It follows that

$$
\lambda_{1} x_{v}=\sum_{w \in V\left(G^{*}\right)} d(v, w) x_{w}=x_{\max } \sum_{w \in V\left(G^{*}\right)} d(v, w)=\left(D_{\max }-\gamma_{n}\right) x_{\max } .
$$

Recall that $D_{\max }-\lambda_{1}=\sigma_{n}$. Therefore we obtain

$$
D_{\max }-\sigma_{n}=\lambda_{1}=\left(D_{\max }-\gamma_{n}\right) \cdot \frac{x_{\max }}{x_{\min }}=\frac{D_{\max }-\gamma_{n}}{1-\sigma_{n}},
$$

which implies that

$$
D_{\max }=\sigma_{n}+\frac{\gamma_{n}}{\sigma_{n}}-1=n+\gamma_{n}-1 .
$$

Hence, $D_{\min }=D_{\max }-\gamma_{n}=n-1$, completing the proof of this claim.

Now, we continue our proof. Since $D_{\min }=n-1$, we get $d(v)=n-1$, which yields that the diameter of $G^{*}$ is two. As a consequence, for each vertex $w \in V\left(G^{*}\right)$ we have

$$
D_{w}=\sum_{z \in V\left(G^{*}\right)} d(w, z)=d(w)+2(n-1-d(w))=2(n-1)-d(w) .
$$

Therefore, for each vertex $w \in V\left(G^{*}\right) \backslash\{v\}$ we get

$$
d(w)=2(n-1)-D_{w}=n-1-\gamma_{n}= \begin{cases}n-2, & \text { if } n \text { is odd } \\ n-3, & \text { if } n \text { is even }\end{cases}
$$

Hence, $G^{*} \cong K_{1,2, \ldots, 2}$ if $n$ is odd, and $G^{*}$ is isomorphic to some $(n-4)$-DVDR graph if $n$ is even. 


\section{Concluding remarks}

In Theorem 1.1 we show that the extremal graph is an $(n-4)$-DVDR graph for even $n$. For any $(n-4)$-DVDR graph $G$ with distinguished vertex $v$, it is clear that the complement of $G-v$ is the disjoint union of some cycles. In addition, by simple algebra, we find that

$$
\sigma(G)=\frac{n+2-\sqrt{n^{2}+4 n-4}}{2}>\frac{1}{n / 2+1}>\frac{1}{n+1} .
$$

Let $H$ be an $r$-uniform hypergraph (i.e., a family of some $r$-element subsets of a set). The shadow graph of $H$ is the simple graph $\partial(H)$ on the same vertex set as $H$, where two vertices are adjacent if they are covered by at least one edge of $H$. Similarly, we can define the distance matrix for connected hypergraphs and non-transmission-regular hypergraphs. It is clear that $H$ and $\partial(H)$ have the same distance matrix for any connected uniform hypergraph $H$. Hence, we immediately obtain the following generalized results from Theorem 1.1.

Theorem 3.1. Let $H$ be a connected non-transmission-regular uniform hypergraph of order $n$.

(1) If $n$ is odd, then

$$
D_{\max }(H)-\lambda_{1}(H) \geq \frac{n+1-\sqrt{(n-1)(n+3)}}{2} .
$$

Equality holds if and only if $\partial(H) \cong K_{1,2, \ldots, 2}$.

(2) If $n$ is even, then

$$
D_{\max }(H)-\lambda_{1}(H) \geq \frac{n+2-\sqrt{n^{2}+4 n-4}}{2} .
$$

Equality holds if and only if $\partial(H)$ is isomorphic to some $(n-4)-D V D R$ graph.

It is worth noting that the extremal hypergraphs in the above theorem are not unique for uniformity at least three. Let $H_{1}$ and $H_{2}$ be 3-uniform hypergraphs on the same vertex set $\{1,2, \ldots, 7\}$, whose edge sets are $E_{1}$ and $E_{2}$, respectively, where

$$
\begin{aligned}
& E_{1}=\{\{1,2,6\},\{1,2,7\},\{1,3,5\},\{1,3,7\},\{1,4,5\},\{1,4,6\},\{2,3,4\},\{5,6,7\}\}, \\
& E_{2}=\{\{1,3,5\},\{1,4,6\},\{1,6,7\},\{2,3,6\},\{2,3,7\},\{2,4,5\},\{4,5,7\}\} .
\end{aligned}
$$

It is clear that $H_{1}$ and $H_{2}$ have the same shadow graph $K_{1,2,2,2}$, while $H_{1}$ is not isomorphic to $\mathrm{H}_{2}$.

\section{References}

[1] F. Atik, P. Panigrahi, On the distance and distance signless Laplacian eigenvalues of graphs and the smallest Gersgorin disc, Electron. J. Linear Algebra, 34: 191-204, 2018.

[2] A. Bondy, M.R. Murty, Graph Theory, Springer-Verlag, London, 2008.

[3] S. Liu, J. Shu, J. Xue, On the largest distance (signless Laplacian) eigenvalue of nontransmission-regular graphs, Electron. J. Linear Algebra, 34: 459-471, 2018. 